\title{
Adaptive Hybrid Precoding for Multiuser Massive MIMO
}

\author{
Xudong Zhu, Zhaocheng Wang, Linglong Dai, and Qi Wang
}

\begin{abstract}
Hybrid precoding (HP) is widely utilized in millimeter wave-based massive MIMO systems with significantly reduced radio frequency (RF) chains, but it requires a large number of analog phase shifters (APSs) and RF adders to realize the connection between RF chains and antenna elements. In this letter, an adaptive hybrid precoding (AHP) is proposed to approach the performance of the conventional HP with reduced complexity. Different from the conventional HP where each antenna is connected to all RF chains through APSs and RF adders, the proposed AHP connects each antenna with only one RF chain through an adaptive connection network. This adaptive connection network and the phases of APSs are jointly designed, which is formulated as an optimization problem to maximize the users' average downlink achievable rate. Moreover, a multiuser adaptive analog precoding (MU-AAP) algorithm is proposed to provide a near-optimal solution to this joint-design problem. Simulation results verify the performance gain of the proposed AHP in typical multiuser massive MIMO scenarios.
\end{abstract}

Index Terms-Massive MIMO, millimeter wave, hybrid precoding, adaptive connection, analog phase shifter.

\section{INTRODUCTION}

M ASSIVE multiple-input multiple-output (MIMO) has been recently investigated to meet the exponential increase of mobile traffic in millimeter wave (mmWave) wireless systems [1]-[3], whereby a base station (BS) equipped with a large number of antennas serves a set of users simultaneously. Asymptotic analysis based on random matrix theory [2] demonstrates that simple linear precoding schemes, such as matched-filter (MF) and zero-forcing (ZF), are virtually optimal and comparable to nonlinear precoding schemes in massive MIMO systems. However, the full digital baseband precoding (FD-BP) scheme [1], [2] requires the same number of radio frequency (RF) chains (including up-converters, digital-to-analog converters, mixers, power amplifiers, and so on) as the number of antenna elements, which leads to extreme high hardware cost in massive MIMO scenarios.

The reduction of the hardware cost has been widely studied in the literature [4]-[9]. The hybrid precoding (HP) [4]-[6] involving both digital and analog processings is able to reduce

Manuscript received December 14, 2015; revised January 17, 2016; accepted February 16, 2016. Date of publication February 19, 2016; date of current version April 7, 2016. This work was supported in part by the National High Technology Research and Development Program of China (Grant 2014AA01A704), in part by the National Natural Science Foundation of China (Grant 61571267), in part by the Beijing Natural Science Foundation (Grant 4142027), in part by Shenzhen Peacock Plan (no. 1108170036003286), and in part by the Shenzhen Visible Light Communication System Key Laboratory (ZDSYS20140512114229398). The associate editor coordinating the review of this paper and approving it for publication was A. Ikhlef.

The authors are with Tsinghua National Laboratory, Information Science and Technology (TNList), Department of Electronic Engineering, Tsinghua University, Beijing 100084, China (e-mail: zhuxd12@mails.tsinghua.edu.cn; zcwang@tsinghua.edu.cn; daill@tsinghua.edu.cn; qiwang11@mails.tsinghua. edu.cn).

Digital Object Identifier 10.1109/LCOMM.2016.2532334 the number of RF chains from the number of antenna elements to the number of data streams. Considering multi-user scenarios, the multi-user HP is proposed in [7] to serve a set of users simultaneously, where the number of required RF chains only equals to the number of users. However, all these existing HP methods are based on the conventional architecture, i.e., each RF chain is connected to all antennas through analog phase shifters (APSs) and RF adders. Thus, the number of required APSs is as large as the product of the number of RF chains and the number of antenna elements, which leads to an excessive hardware cost [8]. Moreover, it should be pointed out that RF adders with multiple inputs are complex [8], [9], and the requirement of a large number of such RF adders makes the practical implementation infeasible.

In this letter, an adaptive hybrid precoding (AHP) is proposed to approach the performance of the conventional HP with reduced complexity. Different from the conventional HP where each RF chain is connected to all antennas through APSs and $\mathrm{RF}$ adders, an adaptive connection network is added between APSs and RF chains, and no RF adders are required. Such network is utilized to adaptively match the analog precoding with the downlink (DL) channel to maximize the users' average DL achievable rate. Specifically, the joint-design of the adaptive connection network and the phases of APSs is formulated as an optimization problem, and a multi-user adaptive analog precoding (MU-APP) algorithm is proposed to provide a near-optimal solution for practical implementation. Simulation results verify the effectiveness of the proposed AHP scheme in typical mmWave based multi-user massive MIMO scenarios.

\section{SYSTEM MODEL}

As shown in Fig. 1 (a), we consider the HP based DL transmission of a multi-user massive MIMO system consisting of a BS with $M$ antennas and $K$ single-antenna users [1][3]. Generally, the received DL signal at all $K$ users can be represented as

$$
\mathbf{y}=\mathbf{H}^{T} \mathbf{F W x}+\mathbf{n},
$$

where $\mathbf{H}=\left[\mathbf{h}_{1} \mathbf{h}_{2} \cdots \mathbf{h}_{K}\right] \in \mathbb{C}^{M \times K}$ denotes the DL channel matrix between the BS and all $K$ users, $\mathbf{F}=\left[\mathbf{f}_{1} \mathbf{f}_{2} \cdots \mathbf{f}_{K}\right] \in \mathbb{C}^{M \times K}$ denotes the analog precoding matrix, $\mathbf{W}=\left[\mathbf{w}_{1} \mathbf{w}_{2} \cdots \mathbf{w}_{K}\right] \in \mathbb{C}^{K \times K}$ denotes the digital precoding matrix, $\mathbf{x} \in \mathbb{C}^{K \times 1}$ denotes the transmit symbol vector with $\mathbb{E}_{\mathbf{X}}\left\{\mathbf{x} \mathbf{x}^{H}\right\}=\frac{P}{K} \mathbf{I}_{K}$ where $P$ is the transmit power at the $\mathrm{BS}, \mathbf{n}=\left[n_{1} n_{2} \cdots n_{K}\right]^{T}$ denotes the additive white Gaussian noise vector, i.e., $n_{k} \sim \mathcal{C} \mathcal{N}\left(0, \sigma_{n, k}^{2}\right)$ at the $k$-th user. To keep the total transmit power unchanged, the digital precoding matrix W should be normalized to satisfy $\|\mathbf{F W}\|_{\mathrm{F}}^{2}=K$, where $\|\cdot\|_{\mathrm{F}}$ denotes the Frobenius norm.

Based on the received uplink pilot signal, the BS is able to obtain the knowledge of channel matrix $\mathbf{H}$ [1]-[3]. After that, variant precoding algorithms [4]-[9] could be utilized to calculate $\mathbf{F}$ and $\mathbf{W}$ for DL transmission. Hence, the DL signalto-interference-plus-noise-ratio (SINR) at the $k$-th user can be 


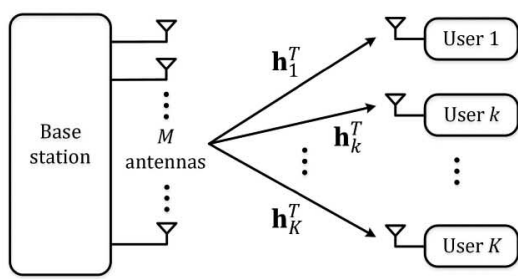

(a)

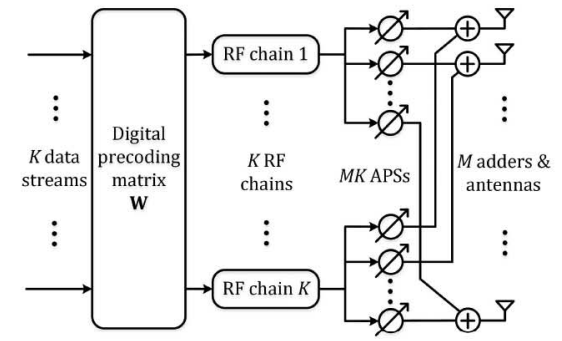

(b)

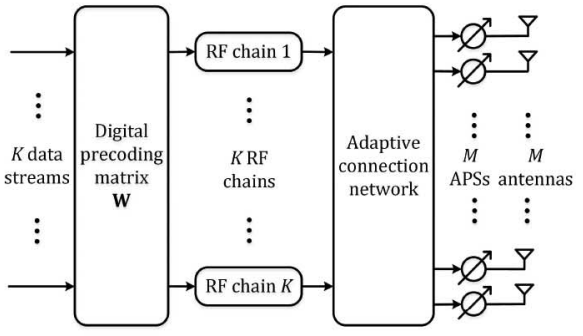

(c)

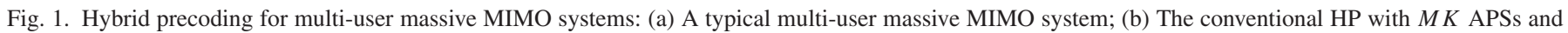
$M$ RF adders; (c) The proposed AHP with an adaptive connection network and $M$ APSs.

represented as

$$
\operatorname{SINR}_{k}=\frac{\frac{P}{K}\left|\mathbf{h}_{k}^{T} \mathbf{F} \mathbf{w}_{k}\right|^{2}}{\sigma_{n, k}^{2}+\sum_{i \neq k} \frac{P}{K}\left|\mathbf{h}_{k}^{T} \mathbf{F} \mathbf{w}_{i}\right|^{2}}
$$

and the corresponding achievable rate of the $k$-th user can be represented as $R_{k}=\mathbb{E}_{\mathbf{H}}\left\{\log _{2}\left(1+\operatorname{SINR}_{k}\right)\right\}$ [4]-[6].

Unlike the digital precoding matrix $\mathbf{W}$, whose elements can be modified in both amplitude and phase, only quantized phase changes can be applied to the analog precoding matrix $\mathbf{F}$ due to the practical constraints of APSs [7]-[9]. By considering $B$ bits quantized APSs, the $j$-th element in $\mathbf{f}_{k}=\left[f_{k, 1} f_{k, 2} \cdots f_{k, M}\right]^{T}$ can be represented as

$$
f_{k, j} \in\{0\} \cup \Omega_{B}, \Omega_{B}=\left\{e^{j \frac{2 \pi n}{2^{B}}}: n=0,1, \cdots, 2^{B}-1\right\},
$$

where $f_{k, j}=0$ denotes that there is no APS between the $j$-th antenna and the $k$-th RF chain.

\section{Proposed Hybrid PRECOding}

In this section, we firstly introduce the architecture of the conventional HP, which requires as much as $M K$ APSs and $M$ $\mathrm{RF}$ adders. Then, the AHP is proposed to approach the performance of the conventional HP with reduced complexity, and the adaptive connection network and the APSs are jointly designed, which is formulated as an optimization problem. After that, a greedy algorithm is derived to provide a near-optimal solution to this optimization problem, together with the analysis of the hardware cost and the computational complexity.

\section{A. Conventional Hybrid Precoding}

As shown in Fig. 1 (b), the widely studied conventional HP is able to approach the precoding performance of the FD-BP [4]-[7], where each antenna is connected to all $K$ RF chains through $K$ APSs and a RF adder. However, the requirement of total $M K$ APSs and $M$ RF adders makes the conventional HP infeasible for practical implementation [7]-[9].

To maximize the users' average DL achievable rate under the constraint of (3), we have [5]-[8]

$$
\mathcal{P}_{\mathrm{HP}}: \underset{\mathbf{F}, \mathbf{W}}{\arg \max }\left\{\sum_{k=1}^{K} R_{k}:(3)\right\} \text {. }
$$

Instead of directly solving this optimization problem $\mathcal{P}_{\mathrm{HP}}$, various greedy algorithms with low complexity are proposed to provide suboptimal solutions [6]-[9]. The core idea of these existing greedy algorithms is to divide the HP design into two step [6]-[9], i.e., the analog precoding and the digital precoding.

Specifically, the analog precoding matrix $\mathbf{F}$ is firstly designed according to the DL channel $\mathbf{H}^{T}=\left[\mathbf{h}_{1} \mathbf{h}_{2} \cdots \mathbf{h}_{K}\right]^{T} \in \mathbb{C}^{K \times M}$ or a predefined codebook [6]-[9]. Then, with the equivalent DL channel $\overline{\mathbf{H}}^{T}=\mathbf{H}^{T} \mathbf{F}$, the conventional digital precoding methods can be adopted to eliminate the inter-user interference, e.g., MF precoding or ZF precoding.

\section{B. The Proposed AHP}

In this subsection, the AHP is proposed to approach the performance of the conventional HP with reduced hardware cost. Specifically, an adaptive connection network is added to connect $M$ APSs to $K$ RF chains, which aims to match the analog precoding with the DL channel to improve the users' DL achievable rate, and can be realized by the digital chip with low computational complexity.

As shown in Fig. 1 (c), different from the conventional HP where only the constraint of (3) is considered, there are two more constraints to be added, i.e.,

$$
\sum_{j=1}^{M}\left|f_{k, j}\right|=N, \text { and } \sum_{k=1}^{K}\left|f_{k, j}\right|=1 .
$$

The first constraint of (5) ensures that each RF chain is connected to $N$ antennas through $N$ APSs, where we assume $N=M / K$ is an integer for simplicity. The second constraint of (5) ensures that each antennas is only connected to one RF chain through one APS, and thus no RF adders are required.

By introducing the equivalent DL channel $\overline{\mathbf{H}}^{T}=\mathbf{H}^{T} \mathbf{F}$ $\left(\overline{\mathbf{H}}^{T}=\left[\overline{\mathbf{h}}_{1} \overline{\mathbf{h}}_{2} \cdots \overline{\mathbf{h}}_{K}\right] \in \mathbb{C}^{K \times K}\right)$, the received DL signal at all $K$ users and the DL SINR of the $k$-th user can be represented as

$$
\mathbf{y}=\overline{\mathbf{H}}^{T} \mathbf{W} \mathbf{x}+\mathbf{n},
$$

and

$$
\operatorname{SINR}_{k}=\frac{\frac{P}{K}\left|\overline{\mathbf{h}}_{k}^{T} \mathbf{w}_{k}\right|^{2}}{\sigma_{n, k}^{2}+\sum_{i \neq k} \frac{P}{K}\left|\overline{\mathbf{h}}_{k}^{T} \mathbf{w}_{i}\right|^{2}}
$$

where $\overline{\mathbf{h}}_{k}^{T}$ denotes the equivalent channel vector between the $\mathrm{BS}$ and the $k$-th user. Considering that joint design of $\mathbf{F}$ and $\mathbf{W}$ leads to unimaginable complexity, the proposed AHP scheme utilizes the analog precoding to enhance the users' equivalent channel gain $\overline{\mathbf{H}}^{T}=\mathbf{H}^{T} \mathbf{F}$ and the digital precoding to eliminate the inter-user interference $\sum_{i \neq k} \frac{P}{K}\left|\overline{\mathbf{h}}_{k}^{T} \mathbf{w}_{i}\right|^{2}$ as shown in Eq. (7), respectively. 
1) Analog Precoding Design: By considering the equivalent channel matrix $\overline{\mathbf{H}}^{T} \in \mathbb{C}^{K \times K}$, it is clear that the $k$-th column in $\overline{\mathbf{H}}^{T}$ as $\overline{\mathbf{h}}_{k}^{T}=\mathbf{h}_{k}^{T} \mathbf{F}$ indicates the equivalent channel gain between the BS and the $k$-th user. Hence, by considering the $k$-th user, the analog precoding design $\mathbf{F}$ could be formulated to maximize the $k$-th user's equivalent channel gain as

$$
\mathcal{P}_{\mathrm{AHP}, \mathbf{F}}^{(k)}: \underset{\mathbf{F}}{\arg \max }\left\{\left\|\overline{\mathbf{h}}_{k}^{T}\right\|_{\mathrm{F}}^{2}=\left\|\mathbf{h}_{k}^{T} \mathbf{F}\right\|_{\mathrm{F}}^{2}:(3),(5)\right\} .
$$

It is clear that these optimization problems provide different analog precoding matrices $\mathbf{F}_{k}(1 \leq k \leq K)$ to maximize the corresponding users' equivalent channel gains. However, only one analog precoding matrix $\mathbf{F}$ can be adopted for all $K$ users. To address this issue, the MU-AAP algorithm is proposed in Subsection III-C.

2) Digital Precoding Design: When the analog precoding matrix $\mathbf{F}$ is fixed, the design of the digital precoding matrix actually becomes a conventional MIMO precoding problem to eliminate inter-user interference $\sum_{i \neq k} \frac{P}{K}\left|\overline{\mathbf{h}}_{k}^{T} \mathbf{w}_{i}\right|^{2}$ as shown in Eq. (7). Then, the classical MF and $\mathrm{ZF}$ digital precoding algorithms [1]-[3] could be adopted as

$$
\left\{\begin{array}{l}
\mathbf{W}_{\mathrm{MF}}=\sqrt{\delta_{\mathrm{MF}}} \overline{\mathbf{H}}^{*}, \\
\mathbf{W}_{\mathrm{ZF}}=\sqrt{\delta_{\mathrm{ZF}}}\left(\overline{\mathbf{H}}^{T}\right)^{\dagger},
\end{array}\right.
$$

where $\delta_{\mathrm{MF}}=K /\left\|\mathbf{F} \overline{\mathbf{H}}^{*}\right\|_{\mathrm{F}}^{2}$ and $\delta_{\mathrm{ZF}}=K /\left\|\mathbf{F}\left(\overline{\mathbf{H}}^{T}\right)^{\dagger}\right\|_{\mathrm{F}}^{2}$ are utilized for power normalization.

\section{Multi-User Adaptive Analog Precoding}

As shown in Fig. 1 (c), the analog precoding matrix $\mathbf{F}$ actually consists of the adaptive connection network and $M$ APSs. Specifically, the adaptive connection network determines the locations of the non-zero elements in $\mathbf{F}$, while $M$ APSs determine the phases of non-zero elements.

Based on (8), it is clear that we have $K$ different optimal analog precoding matrix $\mathbf{F}_{k} \in \mathbb{C}^{M \times K}(1 \leq k \leq K)$ for different users to maximize their equivalent DL channels || $\mathbf{h}_{k}^{T} \mathbf{F}_{k} \|_{\mathrm{F}}^{2}$, respectively. However, based on the system model as shown in Fig. 1 (c), all $K$ users have to share the same analog precoding matrix $\mathbf{F}$. Hence, to guarantee the fairness among all $K$ users, we decompose the design of the analog precoding matrix $\mathbf{F}$ into $K$ columns, and the $k$-th column $\mathbf{f}_{k}$ is designed to match the $k$-th user's DL channel, i.e.,

$$
\mathcal{P}_{\mathrm{AHP}, \mathbf{f}_{k}}^{(k)}: \underset{\mathbf{f}_{k}}{\arg \max }\left\{\left|\mathbf{h}_{k}^{T} \mathbf{f}_{k}\right|:(3),(5)\right\} .
$$

Due to the first constraint in (5), i.e., $\sum_{j=1}^{M}\left|f_{k, j}\right|=N$, only $N$ out of all $M$ elements in $\mathbf{h}_{k}$ are effective. Thus, an intuitive idea is to select the element with the largest amplitude in $\mathbf{h}_{k}$ to be effective, i.e.,

$$
j_{0}=\underset{1 \leq j \leq M}{\arg \max }\left\{\left|h_{k, j}\right|: \sum_{k^{\prime}=1}^{K}\left|f_{k^{\prime}, j}\right|=0\right\},
$$

where the constraint $\sum_{k^{\prime}=1}^{K}\left|f_{k^{\prime}, j}\right|=0$ ensures that the $j$-th APS can be only connected to one RF chain. Then, we set the corresponding element in $\mathbf{f}_{k}$ to match $h_{k, j_{0}}$, i.e.,

$$
f_{k, j_{0}}=e^{-j \frac{2 \pi \hat{n}}{2^{B}}}, \hat{n}=\underset{n \in\left\{0,1, \cdots, 2^{B}-1\right\}}{\arg \min }\left|\frac{h_{k, j_{0}}}{\left|h_{k, j_{0}}\right|}-e^{j \frac{2 \pi n}{2^{B}}}\right| .
$$

TABLE I

BASIC Simulation Parameters

\begin{tabular}{l|l}
\hline \hline Number of antennas at BS & $32 \leq M \leq 1024$ \\
\hline Number of users & $K=8$ \\
\hline Quantized bits of APS & $1 \leq B \leq 6$ \\
\hline Cell radius $R$ & $500 \mathrm{~m}$ \\
\hline Average transmit power at BS & $P_{\mathrm{Tx}}=1$ \\
\hline Average noise power at users & $\sigma_{n}^{2}=0.1$ \\
\hline Log normal shadowing fading $\sigma_{\text {shadow }}$ & $8 \mathrm{~dB}$ \\
\hline Path loss exponent $\alpha$ & 3 \\
\hline \hline
\end{tabular}

Algorithm 1. Multi-User Adaptive Analog Precoding

Input: DL channel $\mathbf{H}$, APS quantized bits $B$.

Output: Analog precoding: F.

1: $f_{k, j}=0,1 \leq k \leq K, 1 \leq j \leq M$.

2: for $t=1$ to $N$ do

3: $\quad$ for $k=1$ to $K$ do

4: $\quad j_{0}=\arg \max _{j}\left\{\left|h_{k, j}\right|: \sum_{k^{\prime}=1}^{K}\left|f_{k^{\prime}, j}\right|=0\right\}$.

5: $\hat{n}=\arg \min _{n \in\left\{0,1, \cdots, 2^{B}-1\right\}}\left|\frac{h_{k, j_{0}}}{\left|h_{k, j_{0}}\right|}-e^{j \frac{2 \pi n}{2^{B}}}\right|$.

6: $\quad f_{k, j_{0}}=e^{-j \frac{2 \pi \hat{n}}{2^{B}}}$.

7: end for

8: end for

Intuitively, the phase of the $f_{k, j_{0}}$ is set as the quantized negative phase of the corresponding channel gain $h_{k, j_{0}}$ to enhance the effective channel $\left|\mathbf{h}_{k}^{T} \mathbf{f}_{k}\right|$ [7]. Here we assume that the DL channel knowledge $\mathbf{H}$ has been obtained at the BS for simplicity [5]-[7]. However, it should be pointed out that effective and perfect channel estimation for such hybrid systems is still an open question, though several works have claimed that channel estimation with high accuracy can be obtained by exploiting channel sparse property [4], [10], [11].

It is clear that (11) and (12) can be consequently carried out for $N$ times to provide a near-optimal solution to $\mathcal{P}_{\mathrm{AHP}, \mathbf{f}_{k}}^{(k)}$. When considering all $K$ users, a simple extended method is to carry out this procedure consisting of (11) and (12) for all users in turns. However, it will cause a severe unfairness among users, e.g., (11) is actually non-functional for the last user's channel vector $\mathbf{h}_{K}$ since there are only $N$ indexes left, which satisfy $\sum_{k^{\prime}=1}^{K}\left|f_{k^{\prime}, j}\right|=0$. To address this issue, an improved algorithm named multi-user adaptive analog precoding (MU-AAP) is proposed as indicated in Algorithm 1. Specifically, within each inner loop of the proposed MU-AAP algorithm, (11) will be carried out for all users only once, and this inner loop will be carried out for $N$ times.

Compared with the conventional HP which requires $M K$ APSs and $M$ RF adders, the proposed AHP only requires $M$ APSs and no RF adders. For example, when $M=1024$ and $K=8$ are considered in typical multi-user massive MIMO systems, the proposed AHP is able to reduce the number of APSs from $M K=8192$ to $M=1024$ and save all $M=1024 \mathrm{RF}$ adders. Moreover, the adaptive connection network can be realized by a switch network controlled by the digital chip with low computational complexity.

In addition, based on Algorithm 1, the computational complexity ratio between the proposed MU-AAP scheme and the conventional HP scheme is actually $\frac{M^{2}+M 2^{B}}{M K 2^{B}} \approx \frac{M}{K}>1$ with a 


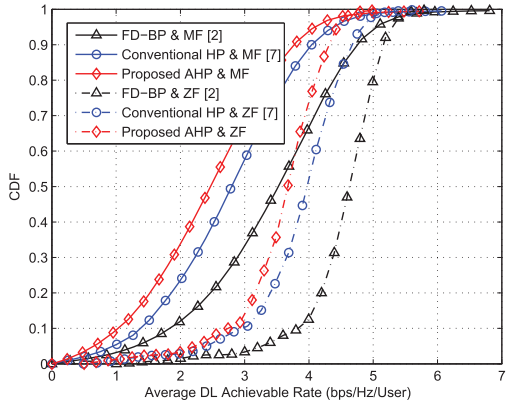

(a)

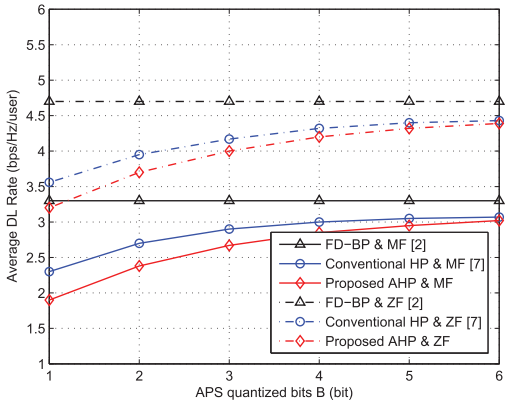

(b)

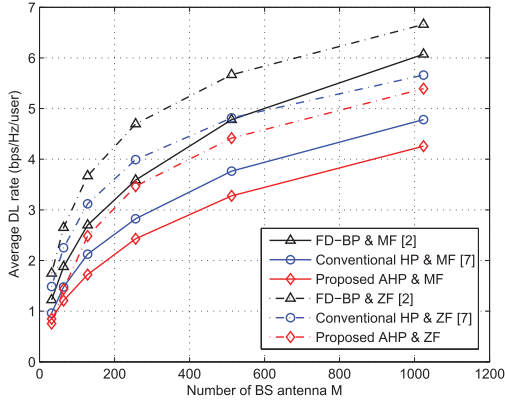

(c)

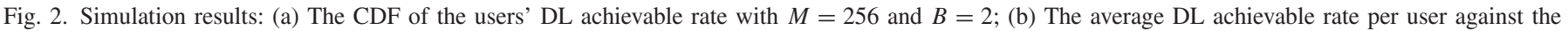
number of quantized bits $B$ with $M=256$; (c) The average DL achievable rate per user against the number of BS antennas $M$ with $B=2$.

large number of BS antennas, which means the computational complexity is slightly increased by the proposed MU-AAP scheme.

\section{Simulation Results}

In this section, we investigate the performance of the proposed AHP through Monte-Carlo simulations. A typical multiuser massive MIMO system is considered [1]-[3], and the basic parameters are list in Table I. As addressed in [1]-[3], the channel vector $\mathbf{h}_{k}$ can be modeled as

$$
\mathbf{h}_{k}=\mathbf{g}_{k} \sqrt{\frac{z_{k}}{\left(r_{k} / R\right)^{\alpha}}},
$$

where $\mathbf{g}_{k} \sim \mathcal{C} \mathcal{N}\left(\mathbf{0}, \mathbf{I}_{M}\right)$ denotes the small-scale fading vector, $z_{k}$ represents the shadow fading and abides a log-normal distribution (i.e., $10 \log _{10}\left(z_{k}\right)$ is zero-mean Gaussian distributed with standard deviation $\left.\sigma_{\text {shadow }}\right), r_{k}$ is the distance between the $k$-th user and the $\mathrm{BS}$, and $R$ denotes the cell radius. The location of users are randomly generated within one cell at each trial (total 500 trials), and the minimal distance between users and the BS is set as $50 \mathrm{~m}$, i.e., $r_{k} \in[50 \mathrm{~m}, R]$.

Fig. 2 (a) plots the cumulative distribution function (CDF) curve of the users' DL achievable rate with $M=256$ and $B=2$. The FD-BP requires $M$ RF chains [2], while the conventional HP requires $K \mathrm{RF}$ chains, $M K$ APSs, and $M \mathrm{RF}$ adders. The conventional $\mathrm{MF}$ and $\mathrm{ZF}$ precoding are adopted in FD-BP, which are the same as those adopted in the digital precoding in HP schemes. For the conventional HP, a low complexity algorithm [7] is adopted, where the phase of each element in $\mathbf{F}$ is set as the quantized negative phase of the corresponding element in the DL channel H. From Fig. 2 (a), it is evident that the proposed AHP, which is realized by the MUAPP algorithm, is able to approach the conventional HP with a small performance gap about $0.3 \mathrm{bps} / \mathrm{Hz}$ with either $\mathrm{MF}$ or $\mathrm{ZF}$ digital precoding.

Fig. 2 (b) shows the average DL achievable rate against the number of quantized bits $B$ of the APS device with $M=256$. It is clear that the average DL achievable rates of both the conventional HP and the proposed AHP can be significantly improved by increasing the quantized bits of the APS device, i.e., $B$. When $B=6$ is considered, the proposed AHP is able to approach the conventional HP with a negligible performance loss, and the performance gap between the proposed AHP and the FD-BP is only about $0.3 \mathrm{bps} / \mathrm{Hz}$ with $\mathrm{MF}$ digital precoding.

Fig. 2 (c) presents the average DL achievable rate per user against the number of BS antennas $M$ with $B=2$. It demonstrates that the precoding performance can be significantly improved by increasing the number of BS antennas for all considered schemes. The proposed AHP is able to approach the conventional HP when $M$ becomes large, and the performance gap to the FD-BP is only about $0.5 \mathrm{bps} / \mathrm{Hz}$ when $M=1024$ is considered.

\section{CONCLUSIONS}

In this letter, we have proposed an adaptive hybrid precoding to approach the performance of the conventional HP with reduced complexity for mmWave based massive MIMO systems. By inserting an adaptive connection network between RF chains and APSs, the analog precoding is able to adaptively match the DL channel to improve the users' average DL achievable rate. Moreover, a multi-user adaptive analog precoding has been proposed to provide a near-optimal solution to the joint-design of the adaptive connection network and the phases of APSs.

\section{REFERENCES}

[1] T. Marzetta, "Noncooperative cellular wireless with unlimited numbers of base station antennas," IEEE Trans. Wireless Commun., vol. 9, no. 11, pp. 3590-3600, Nov. 2010.

[2] F. Rusek et al., "Scaling up MIMO: Opportunities and chanllenges with very large arrays," IEEE Signal Process. Mag., vol. 30, no. 1, pp. 40-60, Jan. 2013.

[3] L. Lu, G. Li, A. Swindlehurst, A. Ashikhmin, and R. Zhang, "An overview of massive MIMO: Benefits and challenges," IEEE J. Sel. Topics Signal Process., vol. 8, no. 5, pp. 742-758, Oct. 2014.

[4] A. Alkhateeb, O. Ayach, G. Leus, and R. Heath, "Channel estimation and hybrid precoding for millimeter wave cellular systems," IEEE J. Sel. Areas Signal Process., vol. 8, no. 5, pp. 831-846, Oct. 2014.

[5] Y. Lee, C. Wang, and Y. Huang, "A hybrid RF/baseband precoding processor based on parallel-index-selection matrix-inversion-bypass simultaneous orthogonal matching pursuit for millimeter wave MIMO systems," IEEE Trans. Signal Process., vol. 63, no. 2, pp. 305-317, Jan. 2015.

[6] F. Khalid and J. Speidel, "Robust hybrid precoding for multiuser MIMO wireless communication systems," IEEE Trans. Wireless Commun., vol. 13 , no. 6 , pp. 3353-3363, Jun. 2014.

[7] L. Liang, W. Xu, and X. Dong, "Low-complexity hybrid precoding in massive multiuser MIMO systems," IEEE Wireless Commun. Lett., vol. 3, no. 6, pp. 653-656, Dec. 2014.

[8] W. Roh, J. Seol, J. Park, B. Lee, J. Lee, Y. Kim, J. Cho, K. Cheun, and F. Aryanfar, "Millimeter-wave precoding as an enabling technology for $5 \mathrm{G}$ cellular communications: Theoretical feasibility and prototype results," IEEE Commun. Mag., vol. 52, no. 2, pp. 106-113, Feb. 2014.

[9] C. Kim, T. Kim, and J. Seol, "Multi-beam transmission diversity with hybrid precoding for MIMO-OFDM systems," IEEE Globecom Workshops (GC Wkshps), Dec. 9-13, 2013, pp. 61-65.

[10] O. Ayach, R. Heath, S. Abu-Surra, S. Rajagopal, and Z. Pi, "Low complexity precoding for large millimeter wave MIMO systems," in Proc. IEEE Int. Commun. Conf. (ICC), Jun. 10-15, 2012, pp. 3724-3729.

[11] O. Ayach, S. Rajagopal, S. Abu-Surra, Z. Pi, and R. Heath, "Spatially sparse precoding in millimeter wave MIMO systems," IEEE Trans. Wireless Commun., vol. 13, no. 3, pp. 1499-1513, Mar. 2014. 